\title{
DIETA DE ORESTIAS AGASSII (CUVIER \& VALENCIENNES, 1846) (TELEOSTEI: CYPRINIDONTIDAE) DEL SALAR DEL HUASCO, NORTE DE CHILE
}

\author{
DIET OF ORESTIAS AGASSII (CUVIER \& VALENCIENNES, 1846) \\ (TELEOSTEI: CYPRINIDONTIDAE) OF “EL HUASCO” SALT LAKE, HIGH \\ ANDES OF NORTHERN CHILE
}

\author{
Jonathan A. Guzmán ${ }^{1,2} \&$ Walter Sielfeld ${ }^{2}$ \\ ${ }^{1}$ Departamento de Zoología, Facultad de Ciencias Naturales y Oceanográficas, Universidad de Concepción. Casilla \\ 160-C. Concepción-Chile. \\ ${ }^{2}$ Laboratorio de Zoología, Departamento de Ciencias del Mar, Universidad Arturo Prat, Casilla 121, Iquique, Chile; \\ jonathanguzman@udec.cl
}

\section{RESUMEN}

Se estudió la dieta de Orestias agassii en tres lagunas asociadas al Salar del Huasco en el altiplano de la I Región. El contenido estomacal presentó; ostracodos, anfípodos, copépodos, moluscos, coleópteros, ácaros y algas macrófitas. Los resultados permiten afirmar que $O$. agassii es un predador carnívoro, que consume una amplia variedad de microcrustaáeos que busca activamente entre la vegetación.

Palabras Clave: Orestias agassii, dieta, comunidades de agua dulce, altiplano sudamericano.

\section{ABSTRACT}

The diet of Orestias agassii from fresh water springs associated to El Huasco Salt Lake were studied. The stomachs analysis indicated depredation on Ostracoda, Cladocera, Amphipoda, Diptera, Copepoda, Mollusca, Coleoptera, Acari and macrofites. It is concluded that $O$. agassii is an active carnivore in freshwater communities of the South-American altiplano.

KeYwords: Orestias agassii, diet, freshwater communities, south-american altiplano.

El genero Orestias Valenciennes, 1893 reúne pequeños peces característicos de los ecosistemas dulceacuícolas de altura (2800 a 4600 m.s.n.m) en Bolivia, Chile y Perú (Arratia 1982, Villwock 1983, Parenti 1984, Villwock \& Thomann 1987, Villwock \& Sienknecht 1993, 1995). En Chile se distribuye en el altiplano con las siguientes especies: $O$. agassii (Valenciennes, 1848), O. piacotensis Vila, 2006, $O$. chungarensis Vila \& Pinto, 1986, O. laucaensis Arratia, 1982, O. parinacotensis Arratia, 1982 у $O$. ascotanensis Parenti, 1984, que constituye el límite sur del género (Salar de Ascotan, II Región) (Jara et al. 1995; Vila, 2006; Vila et al. 2006). Las especies habitan, ríos, lagos, lagunitas y vertientes de salares y bofedales en las regiones I, II y XV (Campos et al. 1998; Dyer 2000), donde entre las macrófitas acuáticas se refugian, alimentan y reproducen (Vila et al. 1999). La depredación y salinización por extracción de agua, son las principales alteraciones a este conjunto (Vila et al. 2006), donde por ejemplo O. ascotanensis, restringida a vertientes con mínimo abastecimiento natural podría verse afectada (Keller \& Soto 1998). Respecto de la conservación, cuatro especies están "En Peligro de Extinción", una 
insuficientemente conocida (O. agassii) y otra $(O$. piacotensis) sin categoría por falta de antecedentes (Vila et al. 2006). Más que la taxonomía, distribución (Arratia 1982; Parenti 1984; Lüssen et al. 2003) y aspectos aislados en la biología de O. laucaensis (Pinto \& Vila 1987) y O. ascotanesis (Jara et al. 1995), el conocimiento del género es deficiente. Esta nota describe la dieta de O.agassii, especie distribuida en los ríos Collacahua, Isluga y vertientes tributarias del Salar del Huasco (3800 m.s.n.m, $68^{\circ} 50^{\prime} \mathrm{W}, 20^{\circ} 15^{\prime} \mathrm{S}$ ), en altiplano de Tarapacá. El estudio abarca tres vertientes del salar: Ermitaño $(n=30)$; Huasco Norte $(n=29)$ y Huasco Lípez $(n=33)$, las que en verano se nutren de las lluvias del "invierno altiplánico". En este salar se desarrolla una considerable biodiversidad, especialmente de aves (Sielfeld et al. 1996), por lo que desde 1981 es considerado un sitio RAMSAR.

Durante diciembre de 2003 se capturó 92 especimenes de $O$. agassii, (Permiso: Subsecretaria de Pesca y Ministerio de Economía) que fueron medidos, (longitud estándar) masados y sexados. Diferencias en la talla estándar por sexos se evaluaron con un análisis de varianza. El contenido estomacal se preservó con alcohol $70^{\circ}$ y fue determinado bajo lupa estereoscópica. La importancia de cada presa se cuantificó con el "Índice de Alimento Principal (MFI), que considera cuatro categorías: ítem esencial (MFI > 75); principal $(51<$ MFI < 75); secundario (26 < MFI $<50$ ) y accesorio (MFI < 26) (Zander 1982). El MFI usa la frecuencia (\%f), abundancia (\%n), y masa (\%g) de las presas (Hyslop 1980), la que se estimó con muestras de referencia. La diversidad trófica se calculó con el índice de Shannon-Weaver $\left(\mathrm{H}^{\prime}\right)$; bajos valores se interpretan como peces estenofágicos y altos como eurifágicos (Berg 1979). Mediante un muestreo epibentónico con un colector tipo Minto (1977) de área fija (13 X $14 \mathrm{~cm}$ ) y cuchillo de corte horizontal, se obtuvo la oferta potencial de presas en Huasco Lípez y Ermitaño.

Las hembras representaron el 51\% $(n=47), 37 \%$ $(\mathrm{n}=34)$ los machos y $12 \% \quad(\mathrm{n}=11) \quad$ los indeterminados. El peso fluctuó entre 0.16 a 10.44 gr., y la longitud estándar entre 21 a $72 \mathrm{~mm}$. No se halló diferencias significativas $(\mathrm{p}=0,519)$ ligadas al sexo, divergiendo con Arratia (1982), sobre el mayor tamaño de las hembras, patrón reportado por Pinto
\& Vila (1987) para O. laucaensis de las lagunas Cotacotani y Río Lauca, y posteriormente Jara et $a l$. (1995) encontraron igual condición para $O$. ascotanensis, además de dimorfismo asociado a la coloración de flancos y aletas (dorsal y anal).

La dieta se presenta en la Tabla I. Según las abundancias, dominan los ostrácodos, copépodos, dípteros (chironomidos), cladóceros, anfípodos, coleópteros (elmidos), ácaros y gasterópodos. Los primeros cuatro cubren el $96,2 \%$, donde copépodos y ostrácodos son los más diversos. Según el MFI, todos son ítemes accesorios, donde crustáceos y dípteros serían más preferidos. Ostracoda presenta el mayor MFI, que se favorece por las valvas y su abundancia, que aumentan su representatividad en la biomasa. La dieta por sexos (MFI) mostró sutiles diferencias, ambos incluyeron algas (Spyrogira sp.), pero hembras más anfípodos y machos más ostrácodos (Figura 1a). Las abundancias de presas por sexo, reflejan que hembras y machos de $O$. agassii consumen más ostrácodos y copépodos, en O. laucaensis las hembras prefieren más anfípodos (Hyalella sp.), y machos más cladóceros (Alonella sp.) (Pinto \& Vila 1987). De manera global, $O$. agassii preda los mismos ítemes que $O$. laucaensis, quien no consume macrófitas, arácnidos y coleópteros (Pinto \& Vila 1987). Los individuos de Huasco Lípez $\left(H^{\prime}=2,1\right)$ y El Ermitaño $\left(H^{\prime}=\right.$ 1,78) fueron eurifágicos y en Huasco Norte (HN) $\left(\mathrm{H}^{\prime}=0,31\right)$ estenofágicos (Tabla II). La oferta en H. Lípez fue de copépodos harpacticoideos (32,6\%), dípteros $(25,4 \%)$, ostrácodos $(23,0 \%)$ y larvas de coleópteros (Elmidae) (6,8\%) (Figura 1b). Contrastados oferta con dieta, se aprecia que Orestias selecciona lo más abundante (crustáceos e insectos). En Ermitaño la oferta fue de moluscos, nematodos (22,2\% cada uno), anélidos, y platelmintos $(21,2 \%$ cada uno) entre otros (Figura 1b). Anfípodos, copépodos, coleópteros y dípteros representan el 1,1\%, sin embargo, en base al MFI, los ítemes menos abundantes, son más consumidos (crustáceos e insectos). En ambas vertientes, los invertebrados blandos no fueron constatados en los estómagos de Orestias, los cuales se capturaron a medio día y contenían abundante alimento sin digerir. Por otra parte; platelmintos, nematodos y otros, habitan el epibentos, donde Orestias no forrajea, siendo de acuerdo a los presentes resultados, un pez carnívoro que busca activamente sus presas entre la vegetación de macrófitas. 
Gayana 73(1), 2009

TABLA I. Composición de la dieta en Orestias agassii en el Salar del Huasco, norte de Chile.

TABLE I. Diet composition of Orestias agassii from Salar del Huasco, Northern Chile.

\begin{tabular}{|c|c|c|c|c|c|}
\hline ITEMES PRESA & $\begin{array}{c}\mathrm{N} \\
\text { individuos }\end{array}$ & $\begin{array}{c}\text { Frecuencia } \\
\%\end{array}$ & $\begin{array}{c}\text { Abundancia } \\
\%\end{array}$ & $\begin{array}{c}\text { Biomasa } \\
\%\end{array}$ & $\begin{array}{c}\mathrm{MFI} \\
\%\end{array}$ \\
\hline MACROFITAS & - & & - & - & - \\
\hline Spyrogira sp. & - & 23 & - & - & - \\
\hline \multicolumn{6}{|l|}{ CRUSTACEA } \\
\hline COPEPODA & 729 & 49 & 26 & 1,5 & 8 \\
\hline Harpacticoidea indeterminado & 470 & 34 & 17 & - & - \\
\hline Cyclopoidea, Eucyclops sp. & 101 & 26 & 4 & - & - \\
\hline Cyclopoidea, Paracyclops sp. & 21 & 14 & 1 & - & - \\
\hline Cyclopoidea, Diacyclops sp. & 84 & 24 & 3 & - & - \\
\hline Cyclopoidea indeterminado & 53 & 25 & 2 & - & - \\
\hline OSTRACODA & 1103 & 72 & 40 & 10,4 & 24 \\
\hline Especie no determinada & 1073 & 72 & 39 & - & - \\
\hline Especie no determinada & 19 & 4 & 1 & - & - \\
\hline Especie no determinada & 11 & 3 & - & - & - \\
\hline CLADOCERA & 383 & 34 & 14 & 13,8 & 18 \\
\hline Leydigia sp. & 298 & 34 & 11 & - & - \\
\hline Chydorus sp. & 70 & 12 & 3 & - & - \\
\hline Cladocera indeterminado & 15 & 9 & 1 & - & - \\
\hline AMPHIPODA & 59 & 12 & 2 & 43,3 & 17 \\
\hline Hyalella sp. & 59 & 12 & 2 & - & - \\
\hline \multicolumn{6}{|l|}{ INSECTA } \\
\hline DIPTERA & 437 & 35 & 16 & 11,8 & 17 \\
\hline Chironomidae indeterminado & 77 & 25 & 3 & - & - \\
\hline Larvas indeterminadas & 360 & 33 & 13 & - & - \\
\hline COLEOPTERA & 17 & 13 & 1 & 4,0 & 5 \\
\hline Elmidae indeterminado & 17 & 13 & 1 & - & - \\
\hline MOLLUSCA & 6 & 4 & 0,2 & 15,1 & 6 \\
\hline \multicolumn{6}{|l|}{ GATROPODA } \\
\hline Taphius sp. & 6 & 4 & 0,2 & - & - \\
\hline ARACHNIDA & 14 & 9 & 1 & - & 0,4 \\
\hline \multicolumn{6}{|l|}{ ACARI } \\
\hline Oribatidae indeterminado & 14 & 9 & 1 & - & \\
\hline Itemes indeterminados & 10 & 7 & 0,4 & - & - \\
\hline Total de presas & 2.758 & & & & \\
\hline Total de taxa & 18 & & & & \\
\hline Total de estómagos & 92 & & & & \\
\hline
\end{tabular}


Dieta De Orestias Agassii: GuZMÁn, J. \& W. SielfELD

TABLA II. Composición de la dieta en Orestias agassii del Salar del Huasco por vertientes.

TABLE II. Diet composition of Orestias agassii from Salar del Huasco by freshwater springs.

\begin{tabular}{|c|c|c|c|c|c|c|c|c|c|c|c|c|}
\hline \multirow[t]{2}{*}{ Itemes } & \multicolumn{4}{|c|}{ Vertiente Ermitaño } & \multicolumn{4}{|c|}{ Vertiente Huasco Norte } & \multicolumn{4}{|c|}{ Vertiente Huasco Lípez } \\
\hline & $\% \mathrm{~N}$ & $\% \mathrm{G}$ & $\% \mathrm{~F}$ & MFI & $\% \mathrm{~N}$ & $\% \mathrm{G}$ & $\% \mathrm{~F}$ & MFI & $\% \mathrm{~N}$ & $\% \mathrm{G}$ & $\% \mathrm{~F}$ & MFI \\
\hline Macrófitas & & & 40 & & & & 24,3 & & & & & \\
\hline Copépodos & 78,4 & 3,1 & 36,7 & 13,3 & 1,2 & 0,1 & 20,7 & 0,91 & 26,8 & 4,3 & 84,8 & 15,6 \\
\hline Ostrácodoo & 13,3 & 1,8 & 50 & 7,6 & 94,4 & 22,7 & 86,2 & 45,3 & 23,2 & 15,8 & 84,8 & 29,2 \\
\hline Cladóceros & 1,1 & 6,1 & 10 & 0,01 & 0,8 & 0,1 & 6,9 & 0,6 & 19,6 & 4,9 & 81,8 & 15,8 \\
\hline Anfípodos & 1,9 & 9,2 & 13,3 & 14,9 & 0,2 & 3,6 & 3,4 & 2,5 & 2,8 & 14,6 & 18,2 & 12,4 \\
\hline Dípteros & 1,9 & 1,1 & 10 & 24,4 & 0,5 & 0,3 & 3,4 & 0,8 & 22,4 & 52,6 & 84,8 & 53,1 \\
\hline Coleópteros & 3,0 & 14,9 & 10 & 9,8 & & & & & 4,8 & 7,8 & 27,3 & 11,2 \\
\hline Moluscos & & & & & 0,9 & 73,1 & 13,8 & 23,2 & & & & \\
\hline Ácaros & & & & & 2,1 & 0,2 & 27,6 & 1,7 & & & & \\
\hline
\end{tabular}

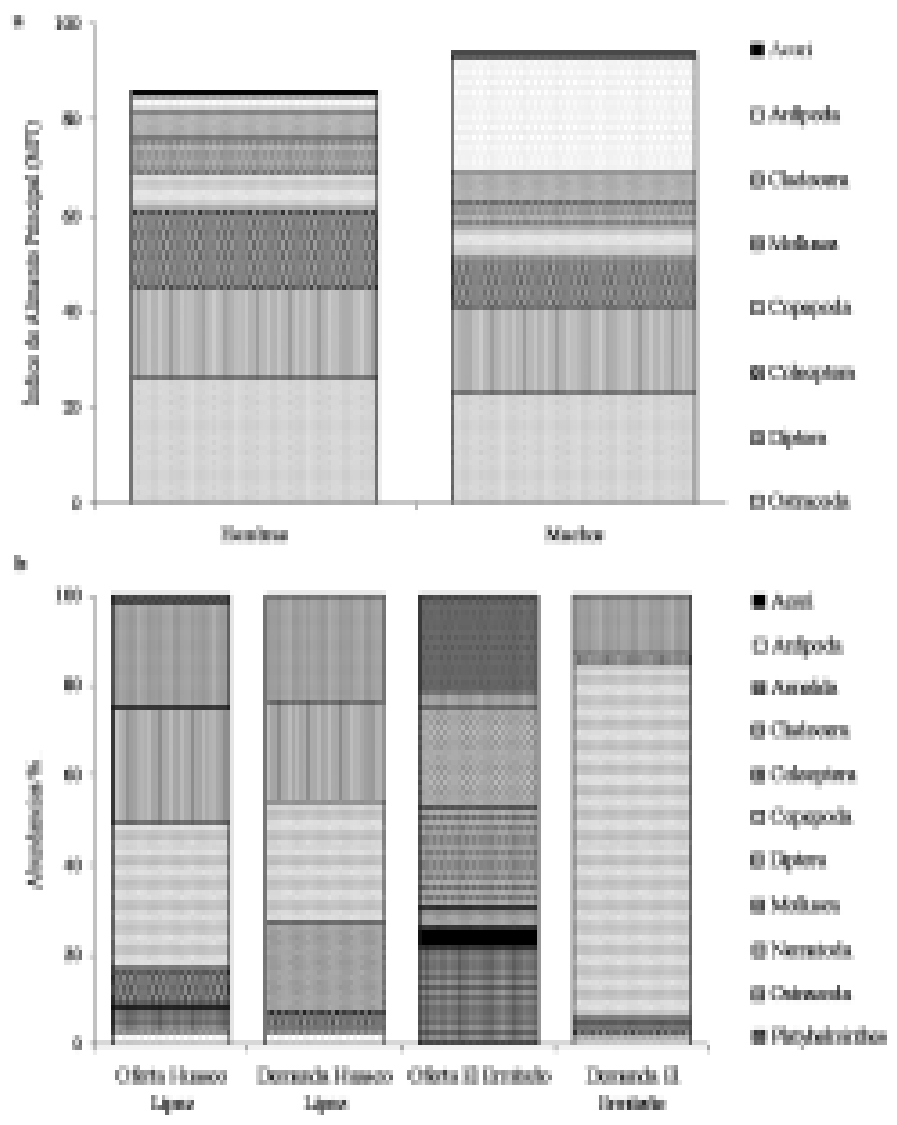

FIGURA 1. Composición de la dieta en Orestias agassii del Salar del Huasco por sexos (a) y por vertientes (b).

FIGURE 1. Diet composition of Orestias agassii from Salar del Huasco by sex (a) and by fresh water springs (b). 


\section{BIBLIOGRAFIA}

Arratia, G. 1982. Peces del altiplano chileno. En: El hombre y los ecosistemas de montaña. Volumen I (Eds. Veloso, A. \& E. Bustos) pp. 93-133. Oficina regional de ciencia y tecnología de la Unesco para America Latina y el Caribe. Montevideo Uruguay.

BERG, J. 1979. Discussion of methods of investigating the food of fishes, with referent to a preliminary study of the prey of Gobiusculus flavescens (Gobiidae). Marine Biology 50: 263-273.

Campos, C. H., G. Dazarola, B. Dyer, L. Fuentes, J. F. Gavilan, L. Huaiquín, G. Martínez, R. Meléndez, G. Pequeño, F. Ponce, V. H. Ruiz, W. Sielfeld, D. Soto, R. Vega \& I. Vila. 1998. Categorías de Conservación de Peces Nativos de Aguas Continentales de Chile. Boletín del Museo Nacional de Historia Natural 47: 101-122.

DYER, B. 2000. Systematic Review and Biogeography of the Freshwater Fishes of Chile. Estudios Oceanológicos 19: 77-98

Habit, E., B. Dyer \& I. Vila. 2006. Estado de conocimiento de los peces dulceacuícolas de Chile. Gayana 70 (1): 100-113

HYsLop, E. J. 1980. Stomach contents analysis - A review of methods and their application. Journal of Fish Biology 17: 411-429.

Jara, F., D. Soto \& R. PaLma. 1995. Reproduction in captivity of the endangered killifish Orestias ascotanensis (Teleostei: Cyprinodontidae). Copeia 1: 226-228.

Lüssen, A., T. M. FAlK \& W. Villwock. 2003. Phylogenetic patterns in populations of Chilean species of the genus Orestias (Teleostei: Cyprinodontidae): results of mitochondrial DNA analysis. Molecular Phylogenetics and Evolution 29: 152-160.

Minto, M.L. 1977. A sampling devise for the invertebrate fauna of aquatic vegetation. Freshwater Biology 7: 425-430.

PinTo, M. \& I. VILA. 1987. Relaciones tróficas y caracteres morfofuncionales de Orestias agassii Arratia 1982 (Pisces, Cyprinodotidae). Anales del Museo de Historia Natural de Valparaíso 18: 77- 84.

Parenti, L. 1984. A taxonomic revision of the Andean killifish genus Orestias (Cyprinodontiformes, Cyprinodontidae). Bulletin of the American
Museum of Natural History 178 (2): 107-214.

Séller, B. \& D. Soto. 1998. Hydrogeologic influences on the preservation of Orestias ascotanensis (Teleostei: Cyprinodontidae), in Salar de Ascotán, northern Chile. Revista Chilena de Historia Natural 71: 147-456.

Sielfeld, W., N. Amado, J. Herreros, R. Peredo \& A. Rebolledo. 1996. La Avifauna del Salar del Huasco: Primera Región Chile. Boletín chileno de Ornitología 3: 17-24

Vila, I., L. Fuentes \& M. Contreras. 1999. Peces Límnicos de Chile. Boletín del Museo Nacional de Historia Natural 48: 61-75.

VILA, I. 2006. A new species of Killififish in the genus Orestias (Teleostei: Cyprinodontidae) from southern High Andes, Chile. Copeia 3: 472-477.

Vila, I., R. Pardo, B. Dyer \& E. Habit. 2006. Peces límnicos; diversidad, origen y estado de conservación. En: Macrófitas y vertebrados de los sistemas límnicos de Chile. (Eds. Vila, I., A. Veloso, R. Schlatter \& C. Ramírez), pp. 73-102. Editorial Universitaria, Santiago de Chile.

VILLWock, W. 1983. El género Orestias y su Evolución en el Altiplano del Perú y Bolivia. Informe Final IX CLAZ. PERU. pp 59-66.

VILLWock, W. \& R. ThomanN. 1987. Mitteilungen über einen neuen Fundort von Orestias Valenciennes, 1839 (Pisces Cyprinodontidae) aus dem nörlichen Altiplano von Chile. Ein Beitrag zur Kenntnis der Verbreitung der Gattung Orestias. Mitteilungen aus dem Hamburgischen Zoologischen Museum und Institut 84: 167 - 174.

Villwock, W. \& U. Sienknecht. 1993. Die Zahnkarpfen der Gattung Orestias Valenciennes, 1893 (Teleostei: Cyprinodontidae) asus dem Asltiplano von Bolivien. Chile und Perú. Mitteilungen aus dem Hamburgischen Zoologischen Museum und Institut 90: 321-362.

VILLWOCK, W. \& U. SienKNEChT. 1995. Intraspezifische Variabilität im Genus Orestias Valenciennes, 1839 (Telesotei: Cyprinodontidae) und zum Problem der Artidentität. Mitteilungen aus dem Hamburgischen Zoologischen Museum und Institut 92: 381-398.

ZANDER, C. D. 1982. Feeding ecology of littoral gobiid and blennioid fish of the Banyuls area (Mediterranean sea). I Main food and trophic dimension of niche and ecotope. Vie Milieu 32:1-10. 